UNIVERSITAS, Volumen 4, Número 1, 2013, 58-70, ISSN 2071-2574

(c) UNAN-León, Editorial Universitaria

EVALUACIÓN DE LA INCERTIDUMBRE EN LA DETERMINACIÓN DE PLOMO EN SANGRE POR VOLTAMPEROMETRÍA DE REDISOLUCIÓN ANÓDICA, APLICANDO EL MODELO DE CALIBRACIÓN PONDERADO DE ADICIÓN PATRÓN GENERADO POR SIMULACIÓN DE MONTE CARLO

\author{
Gustavo Delgado *, Manuel Vanegas, Jairo Salazar.
}

Universidad Nacional Autónoma de Nicaragua, UNAN-LEÓN, Facultad de Ciencias y Tecnología, Departamento de Química, Laboratorio de Análisis de Trazas de Metales Pesados (LATMP), Edificio de Ciencias Básicas, León, Nicaragua. Tel: 5052311 5013, ext: 1132. Fax: (505) 2311 4012.

\title{
(*)e-mail: gusdel53@yahoo.es
}

\section{RESUMEN}

El presente trabajo pretende demostrar que la evaluación de la incertidumbre en la determinación de plomo en sangre por voltamperometría de redisolución anódica, aplicando el análisis de regresión lineal ponderado por adición patrón y generado por simulación de Monte Carlo, tiene mayor congruencia con los resultados experimentales que con los obtenidos por la aplicación del enfoque clásico basado en la ley de la propagación de la incertidumbre. Para el primer caso, se elaboró el programa en Maple 13, basado en el algoritmo obtenido del suplemento 1 de la guía GUM 2008, tomando en cuenta tanto las incertidumbres en las lecturas de la señal instrumental como las involucradas en la preparación de las concentraciones de los calibradores. Los valores característicos del plomo para una muestra de sangre fueron: $3.02 \mu \mathrm{g} / \mathrm{dL}$, con incertidumbre $0.15 \mu \mathrm{g} / \mathrm{dL}$, con un intervalo de cobertura $2.74 \mu \mathrm{g} / \mathrm{dL}$ a $3.29 \mu \mathrm{g} / \mathrm{dL}$. Los resultados obtenidos aplicando la ley de propagación de las incertidumbres a partir del modelo clásico de calibración ponderado, fueron los siguientes: $3.08 \mu \mathrm{g} / \mathrm{dL}$, incertidumbre 0.042 , intervalo de cobertura de 3.00 a $3.16 \mu \mathrm{g} / \mathrm{dL}$. Se observó que hay mayor incertidumbre en el método numérico. No obstante, son coherentes con resultados experimentales de una muestra de sangre.

Palabras claves: Evaluación de la incertidumbre en la determinación de plomo en sangre por simulación de Monte Carlo, determinación de plomo en sangre por voltamperometría de redisolución anódica.

\section{INTRODUCCIÓN}

La voltamperometría de redisolución anódica por diferencial de impulsos (ASV-DP) es una técnica electroquímica de alta sensibilidad para el análisis de metales pesados. Esta técnica consiste en concentrar por electrólisis el ion metálico en un pequeño volumen de una gota de mercurio suspendida $(\mathrm{r}<0.1 \mathrm{~mm})$ a un potencial impuesto; luego el metal se redisuelve por voltamperometría diferencial de impulsos con un barrido de potencial en el sentido anódico. Si las condiciones durante la prelectrólisis se mantienen constantes (velocidad de agitación, tiempo de electrodeposición) la corriente de pico del voltamperograma es proporcional a la concentración de ion metálico en la solución contenida en la celda ${ }^{[1,2]}$. Dependiendo de la matriz y de la naturaleza del ion metálico los límites de detección pueden ser menores que $1 \mathrm{ng} / \mathrm{L}$.

Esta técnica electroanalítica tiene la ventaja de ser utilizada para el análisis trazas tanto de especies inorgánicas como orgánicas ${ }^{[3]}$. Así por ejemplo, para especies metálicas, se ha utilizado en la determinación de plomo y cadmio en análisis de aguas de consumo humano y residuales ${ }^{[4,5]}$ y en la determinación 
de plomo, cadmio, zinc y cobre en muestras de cabello humano de personas en contacto con un medioambiente laboral contaminado ${ }^{[6]}$.

El Laboratorio de Análisis de Trazas de Metales Pesados de la Universidad Nacional Autónoma de Nicaragua-León (LATMPUNAN-León), actualmente utiliza esta técnica para el análisis de plomo en muestras de sangre de personas expuestas a un medio ambiente contaminado con plomo, tales como: fábricas de baterías artesanales, pinturas y talleres de soldaduras. Los niveles de plomo en sangre permitidos según el Center for Deaseases Control $^{[7]}$ son: $<10 \mu \mathrm{g} / \mathrm{dL}$ para menores de 15 años, $<25 \mu \mathrm{g} / \mathrm{dL}$ para mujeres embarazadas y mayores de 16 años, y $<40 \mu \mathrm{g} / \mathrm{dL}$ para varones mayores de 16 años. Con el fin de minimizar la interferencia de matriz y obtener resultados no sesgados, las determinaciones se realizan utilizando el modelo de calibración por adición patrón aplicando el análisis de regresión lineal ponderado ${ }^{[8]}$. La evaluación de la incertidumbre se obtiene mediante la ley de propagación de las incertidumbres ${ }^{[9,10]}$ aplicándola al modelo matemático deducido de la función respuesta o modelo de calibración [8]. No obstante, este método no toma en cuenta las incertidumbres en la preparación de los calibradores o soluciones patrones de calibración. Unmodelodecalibración generado por simulación de Montecarlo ${ }^{[11,12,13]}$, tiene la ventaja de considerar todos los componentes de las incertidumbres involucradas en la construcción de la curva de calibración y por tanto, la incertidumbre de la concentración del analito en la muestra reflejará todos estos componentes.

En el presente trabajo se pretende estimar la incertidumbre en la determinación de plomo en muestras de sangre por ASV-DP a partir de los coeficientes del modelo de calibración ponderado por adición patrón generados por la simulación de Monte Carlo (SMC) y compararla con la obtenida a partir del enfoque clásico de propagación de las incertidumbres.

\section{TEORÍA}

En estudios anteriores ${ }^{[14]}$ se ha evaluado la incertidumbre en la determinación de aflatoxina B1 por HPLC-FD a través de dos vías: a) aplicando la ley de propagación de las incertidumbres, obtenida a partir de la expansión de Taylor tomando en cuenta la derivada de primer orden de la función y despreciando las de orden superior ${ }^{[9]}$; b) por simulación de Monte Carlo ${ }^{[15]}$, en la cual se genera una población de variables aleatorias de entradas con distribuciones específicas (normal, rectangular, triangular, o t); a través de la propagación de estas distribuciones de entradas se genera una población del mesurando con su incertidumbre asociada ${ }^{[13]}$. La figura 1 muestra estos procesos. El cálculo de la concentración $(\mu \mathrm{g} / \mathrm{kg})$ de aflatoxina B1 se efectúo utilizando un modelo matemático que compara la señal analítica (área de pico cromatográfico) de la muestra con la señal obtenida de la solución patrón a un solo nivel de concentración ${ }^{[15]}$.

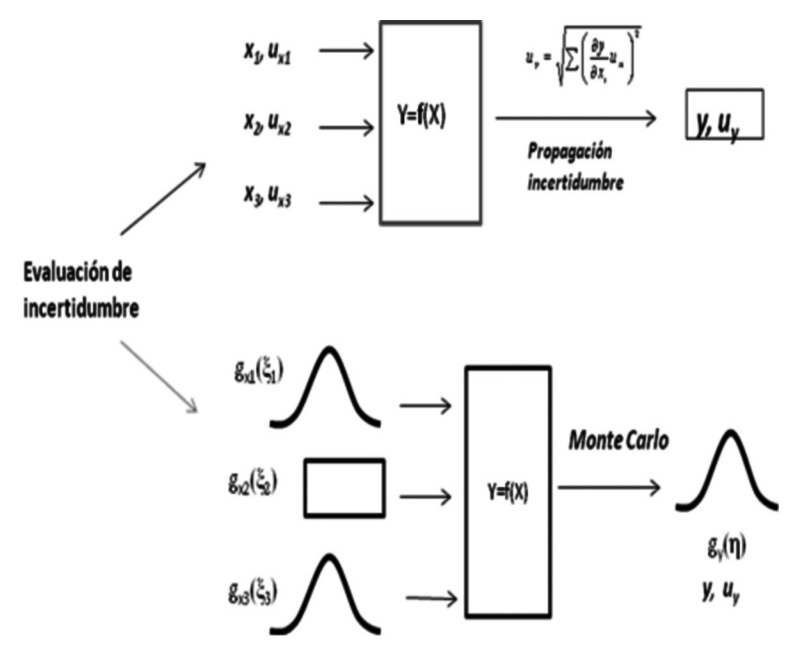

Figura 1. Representación esquemática de la evaluación de la incertidumbre con variables de entradas independientes, calculadas por la propagación de las incertidumbres y por simulación de Montecarlo. 
En el presente trabajo, el contenido de plomo en muestras de sangre se calcula a través del modelo de calibración lineal ponderada por adición patrón generado por simulación de Monte Carlo ${ }^{[13]}$, considerando las distribuciones de la respuesta instrumental y las implicadas en las preparaciones de los calibradores.

A continuación se describen las etapas a seguir en los dos métodos para evaluar la incertidumbre de la medición analítica.

\subsection{Etapas a seguir para evaluar la incertidumbre por método clásico de la propagación de las incertidumbres}

Las varianzas de las corrientes de pico de los voltamperogramas obtenidos por ASV-DP para cada nivel de concentración generalmente no son homogéneas. Las incertidumbres aumentan en función de la concentración de los calibradores. Para verificar la heterocedasticidad de los datos se utiliza una prueba estadística para comparar más de dos varianzas, por ejemplo la prueba de Bartlett o de Cochran ${ }^{[16]}$.

Cuando los datos de la calibración son heterocedásticos, el mejor ajuste del análisis de regresión lineal por mínimos cuadrados se realiza introduciendo los factores de ponderación de cada punto de la recta de calibración en los cálculos de los coeficientes de la regresión. A continuación se describe el principio del método clásico para el cálculo de la incertidumbre de la medición a partir del modelo de calibración por adición patrón obtenido mediante el análisis de regresión lineal ponderado ${ }^{[8,12]}$.

1. Se evalúan los promedios y desviaciones estándares de la respuesta instrumental $\left(\bar{y}_{i}\right.$ ,$s_{i}$ ) para cada nivel de concentración $\left(x_{i}\right)$.
2. Se calculan los factores de ponderación ${ }^{[17]}$ $\left(\mathrm{w}_{\mathrm{i}}=\mathrm{k} / \mathrm{s}_{\mathrm{i}}{ }^{2}\right)$. Siendo $\mathrm{k}$ :

$$
k=\frac{m}{\sum \frac{1}{s_{i}^{2}}}
$$

Donde $m$ es el número de calibradores o niveles de concentración del patrón.

3. Se introducen los factores de ponderación en el cálculo de los coeficientes de regresión:

$$
\begin{gathered}
b_{1}=\frac{\sum w_{i} \sum w_{i} x_{i} y_{i}-\sum w_{i} x_{i} \sum w_{i} y_{i}}{\sum w_{i} \sum w_{i} x_{i}^{2}-\left(\sum w_{i} x_{i}\right)^{2}} \\
b_{0}=\frac{\sum w_{i} y_{i}-b_{1} \sum w_{i} x_{i}}{\sum w_{i}}
\end{gathered}
$$

4. Se calcula la varianza residual y desviaciones estándares del intercepto $\left(\mathrm{s}_{\mathrm{b} 0}\right)$ y la pendiente $\left(\mathrm{s}_{\mathrm{b} 1}\right)$ de acuerdo a las siguientes ecuaciones:

$$
\begin{gathered}
s_{x / y}^{2}=\frac{\sum w_{i}\left(b_{0}+b_{1} x_{i}-y_{i}\right)^{2}}{n-2} \\
s_{b 1}=\sqrt{\frac{s_{x / y}^{2}}{\sum w_{i}\left(x_{i}-\tilde{x}\right)^{2}}} \\
s_{b 0}=\sqrt{\frac{s_{x / y}^{2} \sum w_{i} x_{i}^{2}}{n \sum w_{i}\left(x_{i}-\tilde{x}\right)^{2}}}
\end{gathered}
$$

Donde $\tilde{x}=\frac{\sum w_{i} x_{i}}{n}$

5. Se deduce el modelo matemático que calcula la concentración del analito en la 
muestra $x_{A}$, tomando en cuenta los factores de dilución $f_{i}$ :

$$
x_{A}=\left|\frac{-b_{o}}{b_{1}}\right| \prod f_{i}
$$

6. Se aplica la ley de propagación de las incertidumbres, tomando en cuenta la correlación negativa de los coeficientes de regresión $\left(r_{b 0 b 1}\right), u_{\mathrm{xA}}$ :

$$
u_{x A}=\sqrt{\left(\frac{\partial x_{A}}{\partial b_{0}} u_{b 0}\right)^{2}+\left(\frac{\partial x_{A}}{\partial b_{1}} u_{b_{1}}\right)^{2}+\sum\left(\frac{\partial x_{A}}{\partial f_{1}} u_{f i}\right)^{2}-2\left(\frac{\partial x_{A}}{\partial b_{0}}\right)\left(\frac{\partial x_{A}}{\partial b_{1}}\right) u_{b 0} u_{b 1} r_{b 0, b 1}}
$$

Donde: $u_{i}$ son las incertidumbres de los parámetros de regresión y de los factores de dilución;

$$
r_{b 0 b 1}=\sum x_{i} / \sqrt{m \sum x_{i}^{2}}
$$

\subsection{Etapas para generar el modelo de calibración por SMC ${ }^{[13]}$ :}

1. Se generan $\boldsymbol{N}$ valores aleatorios de la variable $x_{i}$ de los calibradores.

1. Se generan $\boldsymbol{N}$ valores aleatorios de la función respuesta o señal analítica $\left(y_{i}\right)$ para cada nivel de concentración del calibrador.

1. A partir de las variables anteriores se genera una población de $\boldsymbol{N}$ valores de coeficientes de regresión (intercepto $\mathrm{y}$ pendiente), utilizando las ecuaciones 2, 3 .

1. Se genera una población de $\boldsymbol{N}$ valores aleatorios de concentración del analito en la muestra a partir de los $N$ valores absolutos de la razón intercepto/pendiente multiplicada por sus respectivos valores aleatorios de los factores de dilución.

2. Se calcula la concentración del analito en la muestra como el promedio de $\operatorname{los} N$ valores aleatorios generados en 4 .
3. Se calcula la incertidumbre de la concentración del analito en la muestra como la desviación estándar de los $N$ valores de concentración generados en 5 .

4. El intervalo de cobertura se obtiene a partir de la curva de porcentajes acumulados de la función de distribución de probabilidad (CPDF), seleccionando el valor de concentración del límite inferior a 2.5\% cuantiles y el límite superior a 97.5\% cuantiles.

\section{PARTE EXPERIMENTAL}

\subsection{Reactivos}

Se utilizó nitrógeno de alta pureza $>99.999 \%$, proporcionado por un proveedor local. Los estándares de plomo utilizados fueron preparados por dilución de una solución de referencia de plomo de concentración 1000 \pm 0.1 ppm obtenido de Fisher Scientific. El $\mathrm{H}_{2} \mathrm{SO}_{4}$ y $\mathrm{HClO}_{4}$ usados fueron grado trazas de Fisher Scientific. El agua utilizada en las preparaciones de reactivos y estándares fue grado plasma. ${ }^{[17]}$

\subsection{Aparatos}

Se utilizó un analizador voltamperométrico completamente computarizado modelo 797 VA Computrace de Metrohm, controlado por el software Metrodata 797 versión 1.3 de Metrohm; el sistema de tres electrodos utilizados consta de un electrodo de trabajo de mercurio MME (Multi Mode Electrode), tipo de electrodo HMDE, un electrodo de referencia de $\mathrm{Ag} / \mathrm{AgCl} / \mathrm{KCl}(3 \mathrm{~mol} / \mathrm{L}$ ) y un electrodo auxiliar de Pt. Durante el proceso de tratamiento de las muestras de sangre se utilizó un digestor artesanal provisto de un controlador de temperatura conectado a una fuente de poder de $110 \mathrm{~V}$, el cual fue monitoreado con un termómetro digital calibrado, Testo 925, Lenzkirch tipo $\mathrm{K}$, rango de medición -50 a 
$+1000{ }^{\circ} \mathrm{C} \pm 0.7$ a $0.5 \%$. Todas las mediciones de volúmenes necesarias durante este estudio se realizaron con una micropipeta de volumen fijo Eppendorf Research con capacidad de $100 \mu \mathrm{l} \pm 0.3 \%$. Para efectos de dilución de las muestras se utilizó un balón aforado de $10 \mathrm{~mL}$ con tolerancia de $\pm 0.025 \mathrm{ml}$, Pyrex. Los materiales para la toma de sangre venosa, agujas y tubos al vacio conteniendo heparina de sodio como anticoagulante, fueron obtenidos de un proveedor local.

\subsection{Condiciones de operación}

La técnica seleccionada en el equipo es la voltamperometria de redisolución anódica modalidad diferencial de impulsos. El tipo de electrodo seleccionado fue HDME, con un tamaño de gota en la escala 4 y una agitación de $2000 \mathrm{rpm}$. Los rangos de corriente del potenciostato fueron de $100 \mathrm{nA}$ y $1 \mathrm{~mA}$. El tiempo de burbujeo inicial de nitrógeno seleccionado fue de $300 \mathrm{~s}$.
El potencial y el tiempo de deposición fueron de $-0.5 \mathrm{~V}$ y $90 \mathrm{~s}$ respectivamente. El tiempo de equilibrio fue de $20 \mathrm{~s}$. El barrido de potenciales se llevó a cabo desde -0.5 a $-0.15 \mathrm{~V}$ con una amplitud de impulso de $0.05 \mathrm{~V}$ a una velocidad de $0.02 \mathrm{~V} / \mathrm{s}$.

\subsection{Procedimiento}

Se tomaron $200 \square \mathrm{L}$ de sangre total y se digestaron con ácido sulfúrico y ácido perclórico en proporción de 1:4 a un temperatura de $(260 \pm 1){ }^{\circ} \mathrm{C}$. Una vez digestada la muestra fue disuelta en electrolito soporte constituido por una mezcla de $35 \square \mathrm{L}$ de $\mathrm{H}_{2} \mathrm{SO}_{4}$ concentrado y $500 \square \mathrm{L}$ de $\mathrm{HClO}_{4} 1$ $\mathrm{mol} / \mathrm{L}$. Finalmente, se diluyó a un volumen de $10 \mathrm{~mL}$ y se transfirió a la celda electroquímica. Se burbujeó nitrógeno durante $300 \mathrm{~s}$ y se registran 6 réplicas del voltamperograma en las condiciones de operación seleccionadas. Luego se adicionaron 5 volúmenes de $50 \square \mathrm{L}$ de la solución patrón de plomo, registrándose

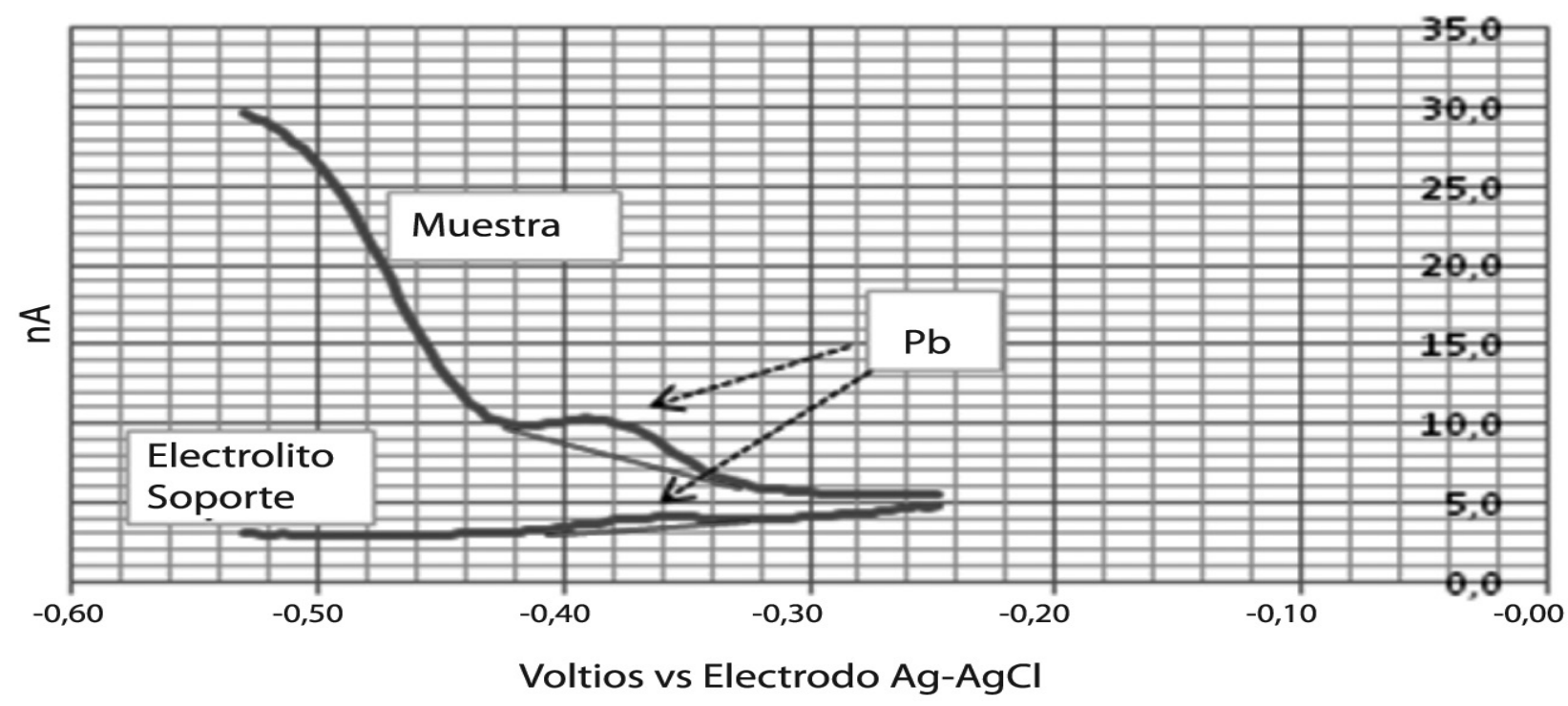

Figura 2. Voltamperogramas de: a) electrolito soporte $\left(\mathrm{HClO}_{4}+\mathrm{H}_{2} \mathrm{SO}_{4}\right.$ ) y b) muestra de sangre disuelta en el electrolito. 
6 réplicas de voltamperogramas por cada adición. Antes de cada réplica del registro del voltamperograma o la adición del patrón de plomo, se burbujeó nitrógeno por $10 \mathrm{~s}$.

\section{RESULTADOS Y DISCUSIÓN}

\subsection{Voltamperogramas obtenidos y verificación del modelo de calibración}

En primer lugar se obtuvo el voltamperograma del electrolito soporte (medio ácido perclórico y sulfúrico) y después el de la muestra de sangre en la solución del electrolito soporte. Los resultados se presentan en la Figura 2. Se puede observar claramente que la línea de base del electrolito soporte es muy diferente a la que presenta la solución muestra, lo que indica que hay un efecto de matriz. Así mismo, se observa que el electrolito soporte presenta un pequeño pico correspondiente a la señal de una impureza de plomo, la cual se sustrajo al voltamperograma obtenido de la muestra.

Los voltamperogramas correspondientes a la solución muestra y las de las adiciones de la solución de referencia de plomo se presentan en la Figura 3. Se puede observar que por cada adición se realizan 6 réplicas del voltamperograma.

Los resultados de las lecturas de corrientes de pico $\left(\boldsymbol{y}_{j}\right)$ en nanoamper (nA) con sus desviaciones estándares para $0.2 \mathrm{~mL}\left(\mathrm{~V}_{\mathrm{x}}\right)$ de la solución muestra contenida en $10 \mathrm{~mL}$ de electrolito soporte $\left(\mathrm{V}_{0}\right)$ y las adiciones de un volumen constante de la solución patrón de plomo (II) $(\mathrm{V}=0.1 \mathrm{~mL})$ de concentración $\mathrm{C}=1000 \square \mathrm{g} / \mathrm{L}$, se presentan en la Tabla 1. Las concentraciones de plomo (II) adicionadas a la muestra se calculan por la ecuación siguiente:

$$
x_{j}=\frac{V_{j} C}{V 0+V_{j}}
$$

Donde $V_{j}=(j-1) V$ con $\mathrm{j}=1 \ldots 6$.

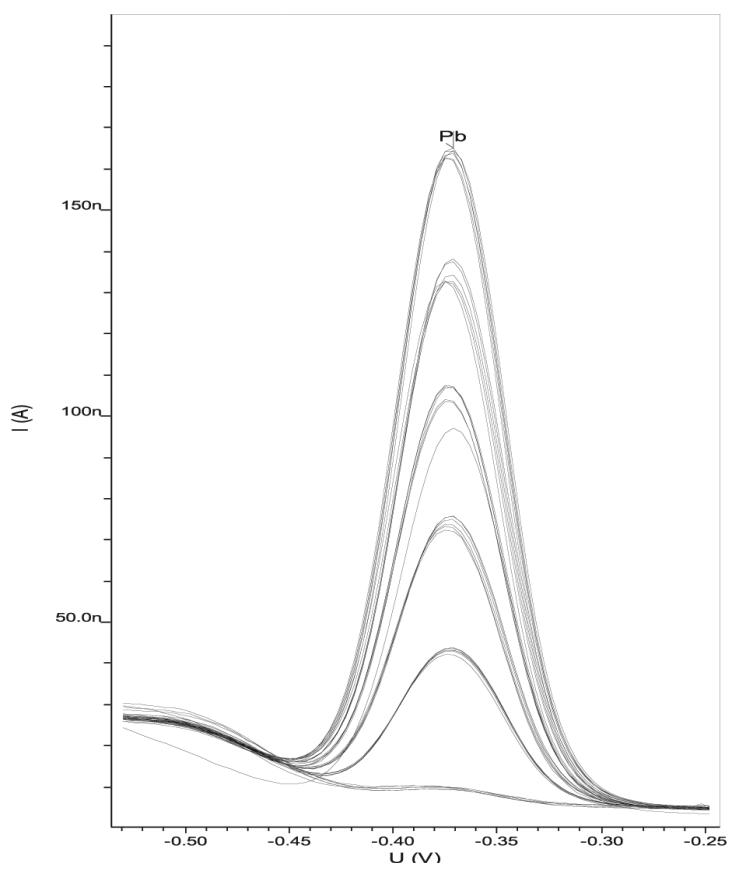

Figura 3. Voltamperogramas (Potencial V - Corriente nA) de redisolución anódica con diferencial de impulsos para una muestra de sangre (curvas azules) y las adiciones del patrón de plomo.

Tabla 1. Volumen en $\mathrm{mL}\left(\mathrm{V}_{\mathrm{j}}\right)$ de la solución patrón de $\mathrm{Pb}$ (II) $1000 \mu \mathrm{g} / \mathrm{L}$ adicionado a $10 \mathrm{~mL}$ de la solución muestra conteniendo $0.2 \mathrm{~mL}$ de sangre, concentración de $\mathrm{Pb}(\mathrm{II})$ adicionado $\left(\mathrm{x}_{\mathrm{j}}\right)$ en $\mu \mathrm{g} / \mathrm{L}$, promedio de 6 réplicas de corrientes de picos $\bar{y}_{j}$ y su desviación estándar $\left(\mathrm{s}_{\mathrm{yj}}\right)$ en $\mathrm{nA}$.

\begin{tabular}{|l|l|l|c|l|}
\hline $\boldsymbol{j}^{\boldsymbol{*}}$ & $\boldsymbol{V}_{\boldsymbol{j}}(\boldsymbol{m L})$ & \multicolumn{1}{|c|}{$\boldsymbol{x}_{\boldsymbol{j}} \boldsymbol{\mu g} / \boldsymbol{L}$} & $\bar{y}_{j}$ & \multicolumn{1}{c|}{$\boldsymbol{s}_{\boldsymbol{y} \boldsymbol{j}}$} \\
\hline 1 & 0 & 0.00 & 1.76 & 0.083 \\
\hline 2 & 0.1 & 9.90 & 33.5 & 0.724 \\
\hline 3 & 0.2 & 19.61 & 63.7 & 1.387 \\
\hline 4 & 0.3 & 29.13 & 93.9 & 2.971 \\
\hline 5 & 0.4 & 38.46 & 123.3 & 2.681 \\
\hline 6 & 0.5 & 47.62 & 152.1 & 1.500 \\
\hline
\end{tabular}

*) Número de calibradores.

\subsection{Verificación del modelo lineal}

Antes de evaluar la incertidumbre en la determinación de plomo en sangre, fue necesario hacer un estudio de la linealidad del 
modelo de calibración. Para ello, se aplicó el análisis de regresión lineal simple (ARLS) por el método de los mínimos cuadrados ${ }^{[8]}$ a los valores correspondientes a $\boldsymbol{x}_{\boldsymbol{j}}$ y los promedios $\bar{y}_{j}$ contenidos en la tabla 1 . Se calcularon los parámetros del modelo $\mathrm{y}=\mathrm{f}(\mathrm{x})$, es decir el intercepto $\left(b_{0}\right)$ y la pendiente $\left(b_{1}\right)$, verificándose la linealidad mediante el cálculo del coeficiente de determinación $\left(\mathrm{r}^{2}\right)$. Los resultados de la regresión se resumen en la Tabla 2.

Tabla 2. Resultados del análisis de regresión lineal

\begin{tabular}{|l|l|l|}
\hline $\boldsymbol{b}_{\boldsymbol{0}}$ & $\boldsymbol{b}_{\boldsymbol{1}}$ & $\boldsymbol{r}^{2}$ \\
\hline 1.962 & 3.154 & 0.99999 \\
\hline
\end{tabular}

De acuerdo al valor de $r^{2}(>0.999)$ se puede inferir que el modelo se ajusta bien a una línea recta.

Una vez demostrada la linealidad de los resultados, se procedió a la evaluación de la incertidumbre en la determinación de la concentración de plomo en la muestra de sangre, utilizando los métodos descritos anteriormente.

\subsection{Evaluación de la incertidumbre por SMC}

A continuación se presenta la implementación de las etapas para el cálculo de la incertidumbre en la determinación de plomo en sangre por simulación de Monte Carlo.

\subsubsection{Generación de 10000 valores aleatorios de $\boldsymbol{x}_{i j}$ y $\boldsymbol{y}_{i j}$ (etapas 1 y 2)}

Sea $x_{i j}$ la variable que representa la concentración de cada calibrador, desde $j=1$ hasta $m$ calibradores y desde $i=1$ hasta $N$ valores aleatorios para cada calibrador. El valor de $N$ es $10000{ }^{[19]}$. El valor de $\mathrm{x}_{\mathrm{ij}}$ para cualquier calibrador $j$ se calcula tomando en cuenta los factores de dilución, según la siguiente ecuación:

$$
x_{i j}=\frac{(j-1) V_{i} C_{i}}{V 0_{i}+(j-1) V_{i}}
$$

V es el volumen alícuota de la solución patrón de plomo $(0.100 \mathrm{~mL})$ adicionada a la muestra utilizando una micropipeta con una desviación estándar de calibración $\mathrm{s}_{\mathrm{v}}=0.003 \mathrm{~mL}$. La concentración de plomo de la solución de referencia certificada es $\mathrm{C}=(1000 \pm 1) \mu \mathrm{g} / \mathrm{L}$, la incertidumbre expandida es $a_{\mathrm{C}}=1$. El volumen de la solución muestra V0 fue medido con un balón de aforo de $10 \mathrm{~mL}$ que tiene una tolerancia $a_{\mathrm{v} 0}=0.025 \mathrm{~mL}$ y desviación estándar de calibración $\mathrm{s}_{\mathrm{v} 0}=0.020 \mathrm{~mL}$. La siguiente gráfica (Figura 4) muestra las distribuciones de las variables que conforman a la distribución de $\boldsymbol{x}_{i j}, \mathrm{gx}_{\mathrm{ij}}(\mathrm{n})$.

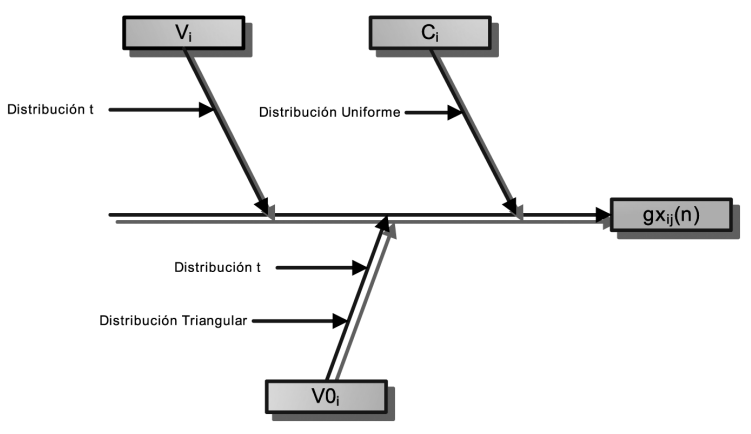

Figura 4. Diagrama Ishikawa mostrando las distribuciones de las variables que caracterizan a $x_{i j}$.

A partir de los valores promedios de las lecturas de corriente de pico simbolizado por $\boldsymbol{l}_{\boldsymbol{j}}\left(\right.$ ver $\overline{\boldsymbol{y}}_{\boldsymbol{j}}$ tabla 1) para cada calibrador $\boldsymbol{j}$ y de los valores de la desviación estándar $\boldsymbol{s} \boldsymbol{y}_{j}$, se pueden obtener $\mathrm{N}$ valores aleatorios de $y_{i j}$ para cada calibrador, considerando que solamente la desviación estándar de las lecturas es el parámetro significativo de dispersión con distribución $t$ que caracteriza a la respuesta instrumental. En la figura 5 se presenta el algoritmo para generar 10000 valores de $x_{i j} \mathrm{y}$ $y_{i j^{*}}$ 

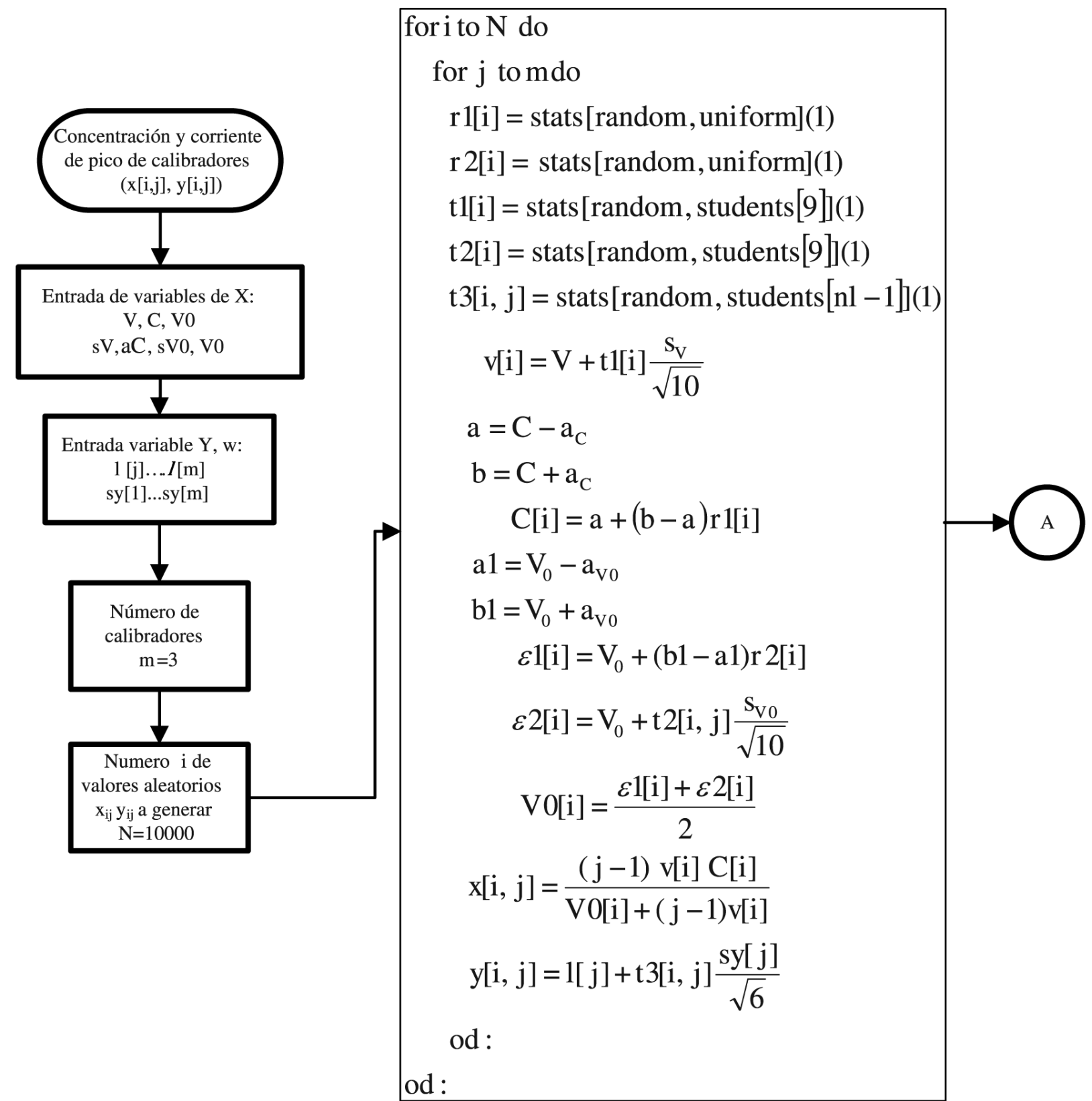

Figura 5. Algoritmo en Maple 13 para el cálculo de 10000 valores aleatorios de $x_{i j}$ y $y_{i j}$.

\subsubsection{Etapas 3 a 7: Cálculo de la concentración} de plomo en la muestra de sangre y su incertidumbre a partir de 10000 valores aleatorios de intercepto y pendiente

Una vez generados los 10000 valores aleatorios de $\boldsymbol{x}_{i j}$ y $\boldsymbol{y}_{i j}$ se utilizan las ecuaciones 2 y 3 para generar 10000 valores aleatorios de pendiente $\left(\boldsymbol{b} \mathbf{1}_{\boldsymbol{i}}\right)$ y de intercepto $\left(\boldsymbol{b} \boldsymbol{0}_{i}\right)$. El valor aleatorio de la concentración del plomo en la muestra de sangre (en $\mu \mathrm{g} / \mathrm{dL}$ ) para cada $\boldsymbol{i}$ se calcula de acuerdo a la ecuación 11 .

$$
c x_{i}=\left|\frac{b 0_{i}}{b 1_{i}}\right|\left(\frac{V 0_{i}+m v_{i}}{10 V x_{i}}\right)
$$


Donde $\mathrm{V}_{\mathrm{i}}$ es el volumen de la solución muestra en la celda, $v_{i}$ es la variable aleatoria del volumen del estándar de plomo adicionado a la celda y $V x_{i}$ es la variable aleatoria del volumen de muestra de sangre (el valor medido es $V x=0.2 \mathrm{~mL}$ ). El valor más probable de la concentración de plomo en la muestra es el promedio de los valores aleatorios de concentración $\boldsymbol{c} \boldsymbol{x}_{\boldsymbol{i}}$ desde $\mathrm{i}=1 \ldots 10000$ y la incertidumbre es la desviación estándar de $\boldsymbol{c} \boldsymbol{x}_{\boldsymbol{i}}$. El algoritmo para este cálculo se puede ver en el algoritmo de la Figura 6. Ejecutando el programa se obtienen los resultados de la tabla 3.

Tabla 3. Resultados del cálculo de la concentración de plomo en sangre con su incertidumbre.

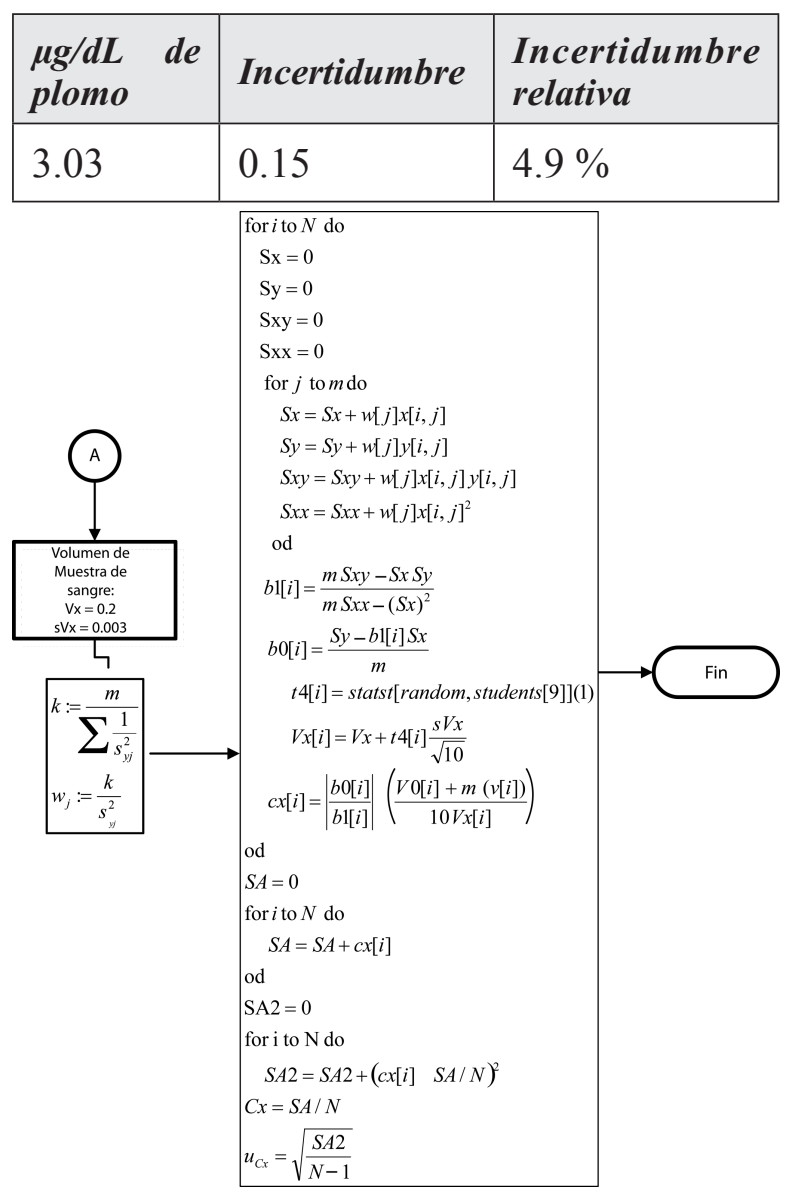

Figura 6. Algoritmo en Maple 13 para el cálculo de la concentración de plomo y su incertidumbre en una muestra de sangre.

\subsection{Estimación del intervalo de cobertura}

El intervalo de cobertura $\left(c_{\text {inf }}\right.$ y $\left.c_{\text {sup }}\right)$ se obtiene a partir de la función de densidad de probabilidad acumulativa (CPDF) de los 10000 valores de $c_{x i}$ generados aleatoriamente. Estos valores fueron exportados a Excel para obtener el histograma y la gráfica de la función de probabilidad acumulativa, seleccionando el valor inferior $\left(\mathrm{c}_{\text {inf }}\right)$ y el superior $\left(\mathrm{c}_{\text {sup }}\right)$ con una probabilidad de 95\%,. La Figura 7 muestra el histograma de los resultados y la curva CPDF, indicando el intervalo de cobertura definido por $2.5 \%$ y $97.5 \%$ cuantiles. Los valores en $\boldsymbol{\mu g} / \boldsymbol{d L}$ son $2.74 \mu \mathrm{g} / \mathrm{dL}$ para $\boldsymbol{c}_{\text {inf }}$ y $3.29 \mu \mathrm{g} / \mathrm{dL}$ para $c_{\text {sup }}$.

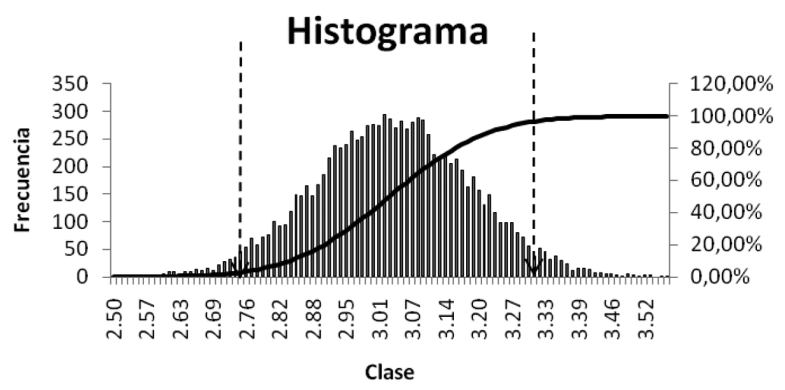

Figura 7. Histograma y gráfico CPDF para los 10000 valores aleatorios de la concentración de plomo en una muestra de sangre. Las flechas verticales de líneas intermitentes representan el intervalo de cobertura: 2.74 y $3.29 \mu \mathrm{g} / \mathrm{dL}$.

El histograma tiene las características de una distribución normal, lo que se verifica con la simetría del sigmoide del CPDF.

\subsection{Evaluación de la incertidumbre por el método clásico de propagación de las incertidumbres}

Aplicando el análisis de regresión lineal ponderado descrito anteriormente mediante las ecuaciones 1 a 8 , se tienen los resultados de la Tabla 4 y el gráfico de la Figura 8. 
Tabla 4. Resultados del análisis de regresión lineal ponderado.

\begin{tabular}{|l|l|l|l|l|l|l|}
\hline $\mathrm{b} 0$ & $\mathrm{~s}_{\mathrm{b} 0}$ & $\mathrm{~b} 1$ & $\mathrm{~s}_{\mathrm{b} 1}$ & $\mathrm{r}^{2}$ & $s_{\text {res }}^{2}$ & $\mathrm{r}_{\mathrm{b} 0 \mathrm{~b} 1}$ \\
\hline 1.837 & 0.03 & 3.16 & 0.00698 & 0.9999 & 0.0053 & 0.801 \\
\hline
\end{tabular}

Para el cálculo de la incertidumbre se utiliza el modelo matemático y la propagación de las incertidumbres (las de tipo A y $\mathrm{B}^{[9]}$ ) de las ecuaciones 7 y 8 . A continuación se detallan las ecuaciones.

La muestra fue diluida solamente una vez, por tanto el factor de dilución $f_{l}$ será:

$$
f_{1}=\frac{V_{0}+6 V}{10 V_{x}}
$$

Donde: $\mathrm{V}_{0}$ es el volumen de la solución muestra contenida en la celda medido en un matraz de aforo de $10 \mathrm{~mL}$ con tolerancia $\pm 0.025 \mathrm{~mL} \mathrm{y}$ desviación estándar de calibración para 10 réplicas $\mathrm{s}_{\mathrm{v} 0}=0.02 ; 6 \mathrm{~V}$ es el volumen total adicionado de la solución patrón de plomo (calibrador 6); Vx es el volumen de muestra de sangre contenido en la solución muestra.

La ecuación 7 se convierte en el siguiente modelo matemático:

$$
c x=\left(\frac{y_{0}-b_{0}}{b_{1}}\right)\left(\frac{V_{0}+6 V}{10 V_{x}}\right)
$$

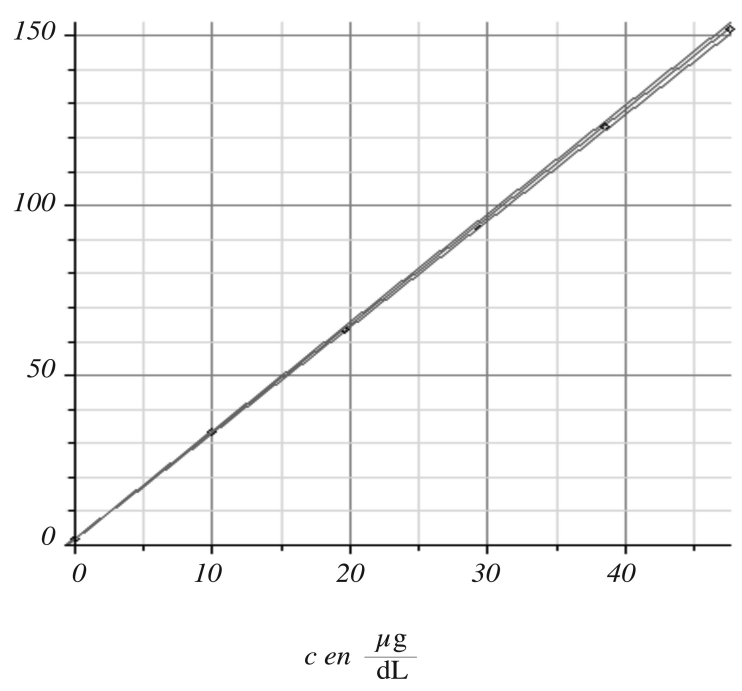

La incertidumbre es ahora expresada por:

$$
u_{c x}=\sqrt{\begin{array}{c}
\left(\frac{\partial c x}{\partial y_{0}} u_{y 0}\right)^{2}+\left(\frac{\partial c x}{\partial b_{0}} u_{b 0}\right)^{2}+\left(\frac{\partial c x}{\partial b_{1}} u_{b 1}\right)^{2}+\left(\frac{\partial c x}{\partial V_{0}} u_{V 0}\right)^{2}+\left(\frac{\partial c x}{\partial V} u_{V}\right)^{2} \\
+\left(\frac{\partial c x}{\partial V_{x}} u_{V x}\right)^{2}-2\left(\frac{\partial c x}{\partial b_{0}}\right)\left(\frac{\partial c x}{\partial b_{1}}\right) u_{b 0} u_{b 1} r_{b 0, b 1}
\end{array}}
$$

Donde $\left(\frac{\partial}{\partial x_{i}} c x\right)$ son los coeficientes de sensibilidad ${ }^{[15]}, x_{i}$ son las variables del modelo, por extrapolación $y_{0}$ tiene el valor de 0.000 con una desviación estándar de la señal del blanco $\boldsymbol{s}_{b l}=0.046$ para 6 réplicas. 
En la tabla 5 y 6 se encuentran la formulación y el cálculo de las incertidumbres de las diferentes variables del modelo (Ecuación 13 y 14).

Tabla 5. Variables del modelo matemático y formulación de las incertidumbres.

\begin{tabular}{|c|c|c|}
\hline Símbolo & Definiciones & $u_{i}$ \\
\hline y0 & Interpolación con el eje $x(0.00)$. & $\begin{array}{l}\mathrm{s}_{\mathrm{ybl}} / \sqrt{6} \\
\text { (desviación estándar del blanco) }\end{array}$ \\
\hline $\mathrm{b}_{0}$ & Intercepto del modelo de calibración & $u_{b 0}=\sqrt{\frac{s_{b 0}^{2}}{6}}$ \\
\hline $\mathrm{b}_{l}$ & Pendiente del modelo de calibración & $u_{b 1}=\sqrt{\frac{s_{b 1}^{2}}{6}}$ \\
\hline $\mathrm{V}_{0}$ & Volumen de la solución muestra (matraz de $10 \mathrm{~mL}$ ) & $u_{V 0}=\sqrt{\frac{s_{V 0}^{2}}{10}+\frac{a_{t o l}^{2}}{6}}$ \\
\hline $\mathrm{V}$ & $\begin{array}{l}\text { Volumen alícuota adicionada a la solución muestra } \\
\text { (micropipeta) }\end{array}$ & $u_{V}=\sqrt{\frac{s_{V}^{2}}{10}}$ \\
\hline $\mathrm{V}_{\mathrm{x}}$ & Volumen de sangre (micropipeta) & $u_{V x}=\sqrt{\frac{s_{V x}^{2}}{10}}$ \\
\hline
\end{tabular}

Tabla 6. Datos e incertidumbres $\boldsymbol{u}_{x i}$ de las variables del modelo matemático. $\boldsymbol{s}_{\boldsymbol{i}}$ es la desviación estándar, $\boldsymbol{a}_{t o l}$ incertidumbre o tolerancia de volumétricos

\begin{tabular}{|l|l|l|l|l|}
\hline Variable $\boldsymbol{x}_{\boldsymbol{i}}$ & Valor/Unidades & $\boldsymbol{s}_{\boldsymbol{i}}$ & $\boldsymbol{a}_{\boldsymbol{t o l}}$ & $\boldsymbol{u}_{\boldsymbol{x} \boldsymbol{i}}$ \\
\hline $\mathrm{y}_{0}$ & 0.000 & 0.046 & & 0.01878 \\
\hline $\mathrm{b}_{0}$ & $5.307 \mathrm{nA}$ & 0.08762 & & 0.03577 \\
\hline $\mathrm{b}$ & $1.665 \mathrm{nA} / \mathrm{mL}$ & 0.01711 & & 0.006985 \\
\hline $\mathrm{V}_{0}$ & $10.00 \mathrm{~mL}$ & 0.02 & 0.025 & 0.001201 \\
\hline $\mathrm{V}$ & $0.100 \mathrm{~mL}$ & 0.003 & & 0.0009487 \\
\hline $\mathrm{V}_{\mathrm{x}}$ & $0.200 \mathrm{~mL}$ & 0.003 & & 0.0009487 \\
\hline
\end{tabular}


Aplicando la ecuación 14 y sustituyendo las variables e incertidumbres de la Tabla 6 , se tiene el valor de incertidumbre asociada a la concentración de plomo en la muestra de sangre, cuyo valor es:

$u_{c x}=0.042$

Considerando un factor de cobertura $\mathrm{k}=2$ para 95.45\% de nivel de confianza, el intervalo de cobertura está dado por:

$\mathrm{C}_{\mathrm{x}} \pm \mathrm{k} \mathrm{u}_{\mathrm{cx}}=3.08 \pm 0.08$

La incertidumbre estándar relativa es: $1.35 \%$.

\subsection{Comparación entre los métodos SMC y el enfoque clásico GUM 1995}

Los resultados de los dos métodos de evaluación de la incertidumbre se resumen en la Tabla 7. Donde se puede ver que el método numérico de simulación de Monte Carlo (SMC) tiene una mayor incertidumbre relativa (IER\%) que el método clásico de propagación de las incertidumbres (GUM): $5.2>1.3$. Los límites inferior y superior del método GUM caen dentro del intervalo del SMC, esto significa que en cuanto a las medias (cx) no hay diferencias significativas. Si se comparan los valores de las incertidumbres estándares relativas y las longitudes del intervalo de cobertura (LIC), se observa que los valores obtenidos por SMC son mayores.

Tabla 7. Resultados de la evaluación de la incertidumbre por el método clásico GUM y el método numérico SMC.

\begin{tabular}{|l|l|l|l|l|l|l|}
\hline Método & $\mathrm{cx}$ & $\mathrm{u}_{\mathrm{cx}}$ & $\mathrm{cx}_{\text {inf }}$ & $\mathrm{cx}_{\text {sup }}$ & IER\% & LIC \\
\hline SMC & 3.03 & 0.15 & 2.74 & 3.29 & 4.9 & 0.55 \\
\hline GUM & 3.08 & 0.042 & 3.00 & 3.16 & 1.3 & 0.16 \\
\hline
\end{tabular}

Estas diferencias se deben a que el método numérico considera no solamente las incertidumbres en la respuesta instrumental (eje $y_{i}$ ) sino que además toma en cuenta las incertidumbres de las concentraciones de los calibradores (eje $x_{i}$ ). Mientras que el método clásico considera solamente la incertidumbre en el eje de las $y_{i}$. El método SMC es más realista por estar basado en las leyes de las probabilidades y sirve para validar otros métodos de cálculo de la incertidumbre ${ }^{[13]}$.

Con el objeto de demostrar cuál de los intervalos está más acorde con los resultados experimentales, se determinó el contenido de plomo en la misma muestra de sangre utilizada en este experimento, registrando 6 voltamperogramas en diferentes días y los resultados en $\mu \mathrm{g} / \mathrm{dL}$ fueron los siguientes:
2.85, 3.05, 2.91, 3.30, 3.01, 2.80. Como se observa, los resultados tienen congruencia con el intervalo de cobertura obtenido por simulación de Monte Carlo.

\section{CONCLUSIONES}

La generación del modelo de calibración ponderado por el método numérico de simulación de Monte Carlo proporciona un estimado más realista de la variabilidad de los resultados de la determinación de plomo en muestras de sangre, dado que toma en cuenta tanto las incertidumbres en la respuesta instrumental como las incertidumbres involucradas en la preparación de las soluciones de calibración. Los resultados se compararon con el análisis de regresión lineal ponderado, aplicando la ley de propagación de la incertidumbre, mostrando 
que éste último presenta mayor precisión en las determinaciones. No obstante, cuando se realizaron 6 veces el análisis de una muestra de sangre, se observó que la dispersión de los resultados está dentro del intervalo de cobertura obtenido por la SMC. Este hecho verifica que las determinaciones voltamperométricas de plomo en muestras de sangre, haciendo uso de la simulación numérica de Monte Carlo descrita en el nuevo suplemento 1 de la guía GUM 2008 para generar los coeficientes de regresión ponderada y en donde se toman en cuenta las dispersiones en la preparación de los calibradores, son más coherentes con los resultados experimentales que los obtenidos por el enfoque clásico de la ley de propagación de la incertidumbre establecida en la guía GUM 1995.

\section{BIBLIOGRAFIA}

1. BARD A. y FULKNER L., (2000), "Electrochemical Methods", 2a edición, John Wiley, NY. Pag. 458.

2. HENZE G., (2003), "Introduction to Polarography and Voltammetry", Metrohm, Suiza, pag. 23.

3. BESSIER P.M., (1994), Analyst, 119, 219.

4. ARROYO L., ALVARADO L y BRAVO S., (1996), Ing. Cienc. Quím., 16, 2, 80-82.

5. CLESCERL L, GREENVERG A, EATON A, (1999), "Standards Methods", American Public Health Association, 20 Edición, NY, pag. 3-52.

6. W, Wasiak, W. Ciszewska, A, Ciszewski, (1996), Analytica Chimica Acta, 335, 201-207.

7. Center for Deasease Control and Prevention, www.cdc.gov/niosh/npg/npgd0368.html. Acceso el 26 de Mayo 2011.

8. Kowalski W, (1998), "Chemometrics" John Wiley, N.Y. pag 132.
9. BIPM, IEC, IFCC, IUPAC, OIML (1995:2008). Guide for to the Expression of Uncertainty in Measurement (GUM), ISO, Ginebra.

10. EURACHEM/CITAC GUIDE, (2000), Quantifying Uncertainty in Analytical Chemistry, 2da edición, UK.

11. OGREN P, DAVIS B y NICK G, (2001), J. Chem. Edu. 78, 6, 827-836.

12. MEIER P y ZUND R, 2000, "Statistical Methods in Analytical Chemistry", John Wiley, N.Y. pag. 166.

13. BIPM, IEC, IFCC, IUPAC, ILAC, ISO, IUPAP y OIML, (2008). "Evaluation of measurements data. Supplement 1 to the Guide for to the Expression of Uncertainty in Measurement (GUM). Propagation of the distributions using a Monte Carlo Method", Paris.

14. DELGADO G. y HERNÁNDEZ N., (2009), "Estimación de la incertidumbre en la determinación de aflatoxina B1 en maní de exportación por HPLC-FD", Universitas UNAN-León, Vol. 3, 1, 5-15.

15. DELGADO G. y HERNÁNDEZ N., (2009), "Cálculo de la incertidumbre por simulación de Montecarlo en la determinación de aflatoxina B1 en maní de exportación por HPLC-FD. Aplicación a la evaluación de la conformidad", Universitas UNAN-León, Vol. 3, 1, 16-26.

16. NEULLY. M. (1998), "Modélisation et estimation des erreurs des mesures", Technique \& Documentation, 2a edición, París, pag. 53.

17. LATMP-PT-01, 2010, "Procedimiento para la determinación de plomo en sangre por Voltamperometría de redisolución anódica por diferencial de impulso (ASV-DP)". Laboratorio de Análisis de Trazas de Metales Pesados (LATMP), UNAN-León. 